\title{
Thermal decomposition of acrylic pressure-sensitive adhesives
}

\author{
Zbigniew Czech*, Robert Pełech, Krzysztof Zych \\ West Pomeranian University of Technology, Institute of Chemical Organic Technology, Szczecin, ul. Putaskiego 10, \\ 70-322 Szczecin, Poland \\ * corresponding author: e-mail: psa_czech@wp.pl
}

The general aim of this article is to review the state of knowledge on pressure-sensitive adhesives (PSAs) and pyrolysis. Recent research data in the field of pyrolysis gas- chromatography (Py-GC) analysis of acrylic PSAs are presented. First, PSA characteristics and applications, pyrolysis (including Py-GC) as an analytical method, and system solutions, are described. The latest scientific achievements in the analysis of thermal degradation products of acrylic PSAs are then presented.

Keywords: acrylic and methacrylic copolymers, pressure-sensitive adhesives, pyrolysis, thermal degradation products, analysis and identification.

\section{PRESSURE-SENSITIVE ADHESIVES (PSAs)}

Today, pressure-sensitive adhesives (PSAs) are widely used in everyday life. PSAs were developed relatively recently (in the latter half of the twentieth century), but, from the beginning, PSAs have been successfully applied in many areas. PSAs are used in self-adhesive tapes, splicing tapes, labels, protective films (in the paper industry), dermal dosage systems for pharmaceutical applications, hydrogels, biomedical electrodes (medical self-adhesive materials), automotive parts assembly (in the automotive industry), toys, electronic circuits, and keyboards (in the electrical industry).

Such a variety of applications results from the many valuable properties of PSAs1, including instantaneous adhesiveness, repeated adhesiveness, tackiness, good aging characteristics, resistance to elevated temperature and plasticizers, exceptional optical clarity (because of polymer compatibility and non-yellowing), and water resistance. Acrylic PSAs show the highest balance of adhesion and cohesion and low (between $-30^{\circ} \mathrm{C}$ to $-70^{\circ} \mathrm{C}$ ) glass transition temperatures $\left(\mathrm{T}_{\mathrm{g}}\right)$.

The great advantage of PSAs is that they stick to almost every surface (with even a little pressure), and physical properties do not change after binding of the two surfaces. No chemical reaction occurs during binding. There are six main families of PSAs (Fig. 1). Acrylic PSAs, in comparison with (for example) polyurethane PSAs, can be formulated in a greater variety, allowing optimization for particular tasks. Acrylic PSAs are typically random copolymers of a long side-chain acrylic moiety (n-butyl

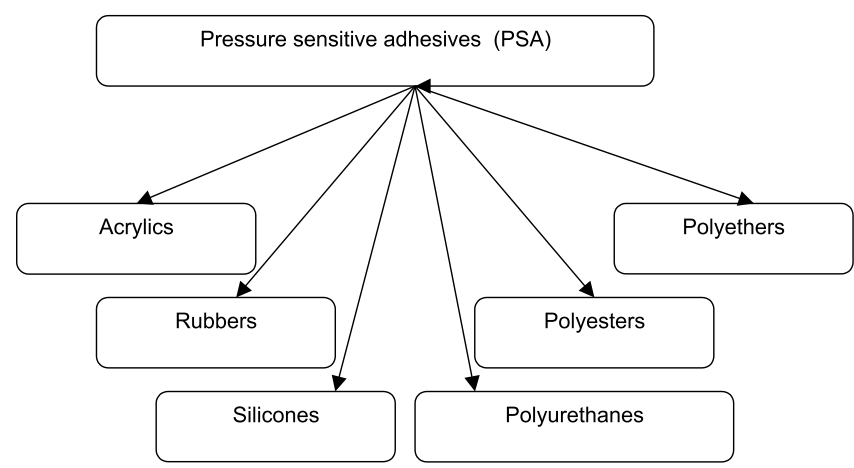

Figure 1. Pressure-sensitive adhesives (PSAs); the various classes acrylate or 2-ethylhexyl acrylate), a short main-chain component (e.g., methyl acrylate; to adjust the $\mathrm{T}_{\mathrm{g}}$ value) and acrylic acid, to improve adhesion and optimize elongational properties $^{2}$. There are various types of acrylic PSA (depending on the solvent used), which can be categorized as solvent-borne, water-borne, and solvent-free.

Relatively few methods of acrylic PSA production have been developed; these include the typical radical thermal polymerization and UV-induced photopolymerization. Recently, new methods to obtain acrylic PSAs have been developed ${ }^{3}$.

\section{PYROLYSIS AND PYROLYSIS GAS CHROMATOGRA- PHY (PY-GC) AS ANALYTICAL METHODS.}

Pyrolysis is a method of thermal degradation of substances at high temperature without contact with oxygen or any other oxidizer. Pyrolysis is performed in the absence of a catalyst. Usually, during pyrolysis, complex compounds in a substance undergo decomposition to simpler compounds of lower molecular weight. Complex materials, after thermal degradation at high temperature, are divided into three groups: the gaseous, liquid, and residue fractions.

The mechanisms of transformation during pyrolysis are often very complex and many studies on pyrolysis have appeared. Pyrolysis is a well-known and successful method used either analytically or to recover monomers from waste polymers. For the last 50 years, many groups of materials have been analyzed by classic pyrolysis and variations thereof ${ }^{4}$. Pyrolysis methods are typically either on-line (where decomposition and analysis are conducted in one device) or off-line (decomposition and analysis proceed using separate systems). Pyrolysis has been successfully employed to manage the waste generated during polymer production, for example to recover monomer from waste polymer. Laboratory research may be successfully extended to a larger scale (a mini-pilot plant). A successful large-scale trial of pyrolysis of filled PMMA, employing a system akin to a laboratory plant, was reported by Kaminsky and Eger ${ }^{5}$.

In the latest work, an increasing tendency toward use of online methods (Py-GC, Py-MS,

Py-GC/MS) may be observed. This is understandable, because by heating a sample in a closed system, in an inert 
gas, and next feeding the pyrolysate directly into a gas chromatograph (Py-GC), it is possible to identify the original unsaturated monomers ${ }^{6-7}$. Py-GC is used to identify and structurally investigate non-volatile organic compounds, usually synthetic polymers and natural organic acid salts, and some other products with more complex structures. The complex pyrograms obtained function as "fingerprints" of the investigated material, in a manner similar to organic compound infra-red (IR) spectra. PyGC is a useful method to identify polymers ${ }^{8}$, particularly rubbers (made of chloroprene, silicone, or polyurethane), to differentiate rubbers (polybutadiene from polyisoprene, or natural from synthetic), to distinguish among industrial adhesives (alkylphenol, coumarone, or terpenephenol), and to analyze similar natural polymers (cotton or animal hair components). Py-GC is useful in the determination of important factors such as molecular weight $\mathbf{M}_{n}$ (which characterizes polymers), via end-group analysis ${ }^{9}$. An advantage of this technique is that no sample preparation is required, but a disadvantage is that not all polymeric material can be used for $\mathrm{M}_{n}$ determination because endgroups are required in any investigated substance. Pyrolysis of polyolefins yields three types of fragments: saturated hydrocarbons, olefins, and diolefins. Wang and colleagues showed that Py-GC was a very useful method for investigating the composition and microstructure of polyolefin copolymers and Py-GC data were in very good agreement with $\left[{ }^{13} \mathrm{C}\right]-\mathrm{NMR}$ results; Py-GC is, in addition, reliable, reproducible, simple, and inexpensive ${ }^{\mathbf{1 0}}$. In several reports on polymer additive analysis by Py-GC, Wang's group described how Py-GC could be coupled with mass spectrometry ${ }^{11-14}$. Plasticizers can be successfully identified on the basis of pyrolysate patterns. The presence of any particular plasticizer is associated with copolymer composition, because the plasticizers exist in an internally modified polymer backbone. The most important advantage of Py-GC plasticizer analysis is that no sample preparation is required and all information can be obtained from one experiment ${ }^{\mathbf{1 1}}$. In some polymers, halogenated organics and non-halogenated phosphate esters (used as flame retardants) are present. These can be qualitatively and quantitatively analyzed by Py-GC and, simultaneously, the polymers are identified. The disadvantage of this method is that with a mixture of organic and inorganic compounds (e.g., a halogen/antimony synergistic pair), it is possible to examine only the organic material. To detect flame retardants, Atomic Emission Detection (AED) run after Py-GC is more effective than Py-GC/MS analysis. In AED, halogen- and phosphorus-containing pyrolysates can be evaluated (flame retardants are identified by peak pattern recognition on an AED-specific element trace) and, in MS, flame retardants are characterized by the specific isotope ratios of chlorine and bromine (thus evaluating only the halogenated portions of the polymers) ${ }^{\mathbf{1 2}}$. Fatty acids (stearic acid and a mixture of hydrocarbons from $\mathrm{C}_{20}$ to $\mathrm{C}_{50}$ or higher) and their related esters, amides, and salts are used as lubricants in polymers. Stearic acid decomposition yields a set of alkenes from $\mathrm{C}_{3}$ to $\mathrm{C}_{17}$ and also, typically, results in aliphatic hydrocarbon chain $\left(\mathrm{C}_{20}\right.$ to $\mathrm{C}_{50}$ ) degradation. The disadvantage of this method is that an auxiliary technique is required to complete the identification of esters, amides, and metallic salts of the stearic acid family. Another disadvantage is the need for pre-separation of the additive package from the parent polymer. The advantages are, of course, simplicity and quick turnaround time. The method is not reliable for all samples, but may be used for screening prior to application of other procedures ${ }^{\mathbf{1 3}}$. Pre-separation of samples is also required in the analysis of a very important class of additives in polymers, namely the antioxidants. The difficulties in identifying antioxidants in polymers lie in the similarities in structure and quantity of antioxidants in various polymers ${ }^{\mathbf{1 4}}$. Py-GC has many immediate applications, particularly in the medical field. Asperger and colleagues studied acryl-based copolymer retarding layers on drug granules using Py-GC to determine if a poly(ethylacrylate-methylmethacrylate) (PEAMMA) copolymer layer would ensure sustained release of an active agent after oral intake of granules. The Py-GC analysis data from kinetic dissolution tests showed that two of six samples displayed unsatisfactory drug release. A conclusion of this work was that Py-GC was useful to determine the quality of drug granules featuring a PEAMMA layer ${ }^{\mathbf{1 5}}$.

\section{LATEST SCIENTIFIC ACHIEVEMENTS IN THERMAL DEGRADATION OF ACRYLIC PSAS.}

Pyrolysis is a significant technique in synthetic polymer research. For over 30 years, development of pyrolysis techniques resulted in new applications in qualitative and quantitative analysis, and structure determination. Application of pyrolysis requires coupling with another technique, which allows the mixture obtained after pyrolysis (the pyrolysate) to be characterized. In research into thermal degradation of acrylic PSAs in the Institute of Chemical Organic Technology of the West Pomeranian University of Technology, Szczecin, both off-line and on-line pyrolysis methods are used. The off-line method employs the system presented in Fig. 2.



Figure 2. Pyrolysis apparatus: 1 - quartz flask, 2 - electric furnace, 3 - condenser, 4 - liquid products collector, 5 - blowndown valve, 6 - septa, 7 - hydraulic valve, 8 - quartz balls, 9 - temperature controller, 10 - thermal insulation 


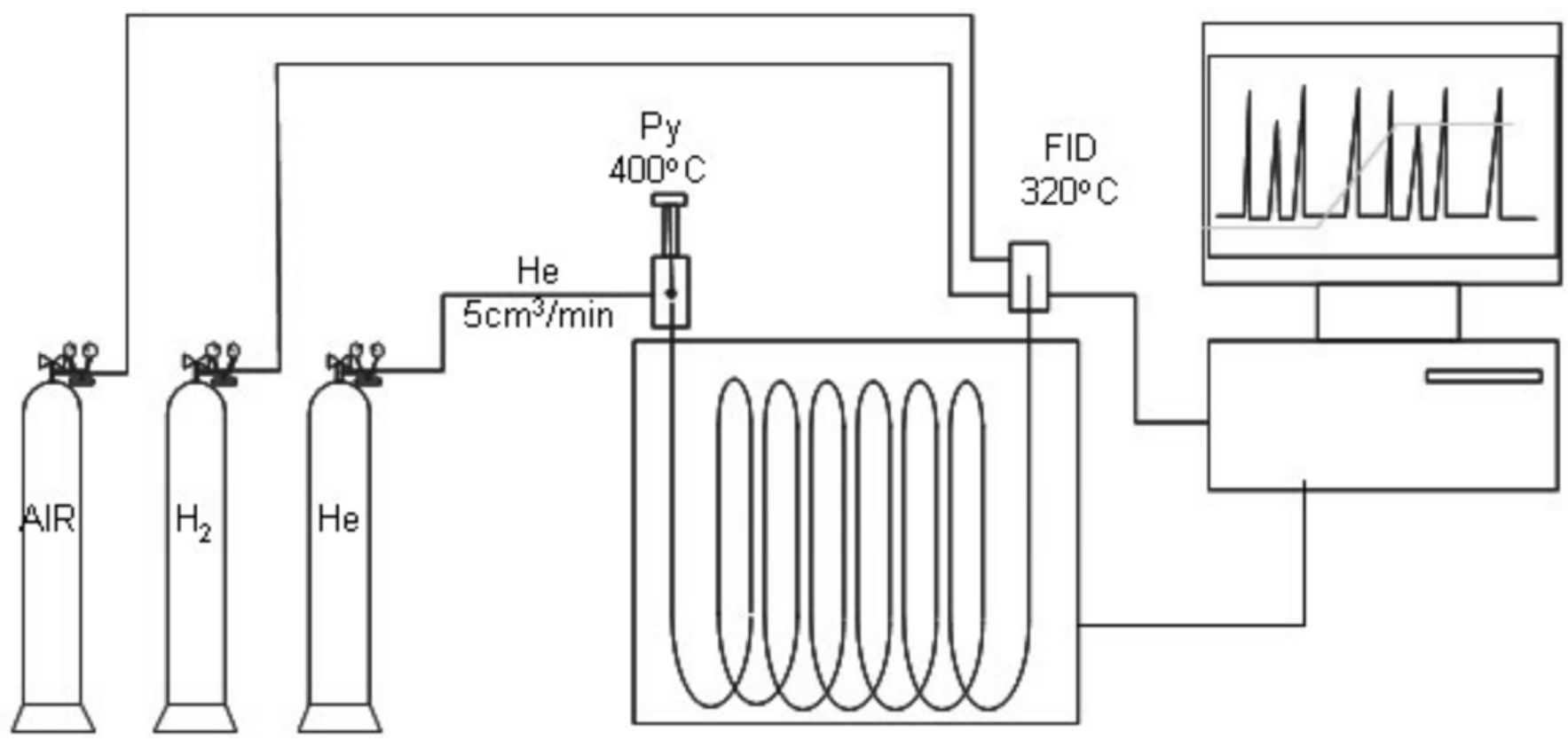

Figure 3. Apparatus for pyrolysis gas chromatography

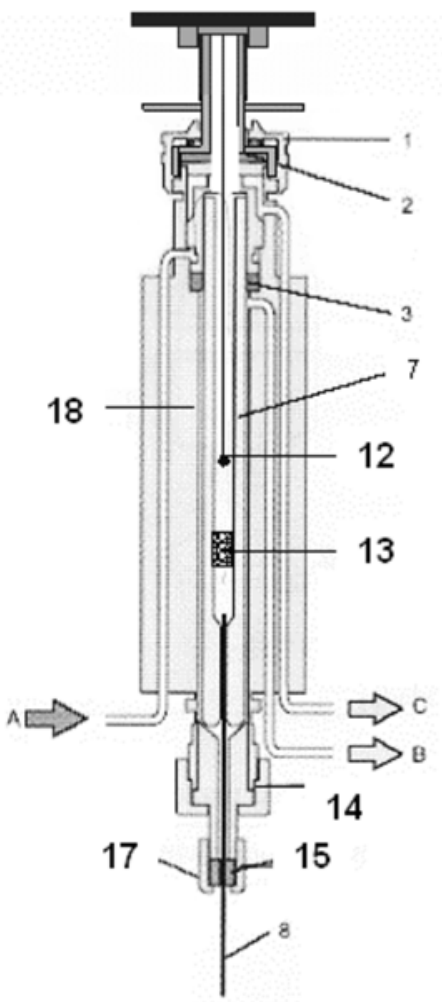

1. Screw with clamping spring

2. Gasket

3. Clamping ring

4. Gasket's support

5. Insert's cover

6. Insert's graphitoidal packing

7. Quartzinsert

8. Nut with packing supporting

glass rod with sample

9. Glass rod

10. Sample insertion line

11. Radiator

12. Sample

13. Glass wool

14. Calmping nut

15. Column's packing

16. Column

17. Nut

18. Heating elements

A - Carrier's gas inlet

$B$ - Blow through line

C - Split stream line



Figure 4. Detailed description of the pyrolyser

Py-GC is a promising and reliable method for thermal degradation analysis of acrylic PSAs. This technique was developed in 1950s, and is a variation on reactive gas chromatography which explores the thermal decomposition of non-volatile substances. Py-GC requires use of a special tip, termed a pyrolyser, instead of a normal feeder ${ }^{7}$. The system is shown in Figs. $3-4$.

In pyrolysis, a gas chromatography flame ionization detector (FID) is usually used. An FID is characterized by a low limit of detection $\left(10-12 \mathrm{~g} / \mathrm{cm}^{3}\right)$ and a wide range of linearity $\left(10^{7}\right)$.

The method is especially useful in the analysis of organic compounds, which are often components of a pyrolysate. During the thermal degradation of acrylic PSAs, products such as carbon dioxide, carbon monoxide, and water may be formed ${ }^{16}$. Such compounds cannot be detected using an FID, but can be detected using a universal conductivity detector (a katharometer TCD). A TCD is characterized by a considerably higher limit of detection $\left(10^{-7}-10^{-8} \mathrm{~g} / \mathrm{cm}^{3}\right)$. In such analysis, a capillary column and high-purity helium carrier gas are usually employed. When comparative detectors (FIDs) are used, it is advisable to conduct the analysis in a two-column system with different column packing. This method considerably increases the certainty of qualitative analysis. However, cross- 
checks using complementary techniques such as NMR, FTIR, MALD-MS, TG, and DSC are also required ${ }^{17}$.

Czech and Pełech showed, in several reports, that PyGC was useful and effective in analyzing the thermal degradation of acrylic PSAs. The pyrolysis of such PSAs (copolymers of acrylic acid and the corresponding acrylate) was conducted in the apparatus described above (Fig. 3) using a two-column system and two temperature programs. During pyrolysis of PSA based on a copolymer of 2-ethylhexyl acrylate (2-EHA) and acrylic acid (AA), the following degradation products were formed: 2ethylhexene-1, 2-ethylhexanol-1, 2-ethylhexyl acrylate and 2-ethylhexyl methacrylate (Fig. 5) ${ }^{9}$. The mechanism of formation of olefin (2-ethylhexene-1) is presented in Fig. 6 . The pyrolysis of a solvent-borne water-soluble copolymer of acrylic acid and butyl acrylate yielded the pyrogram of Fig. 7. The main products were 1-butene, 1-butanol, butyl acrylate, and butyl methacrylate ${ }^{18}$. In the analysis of copolymers of acrylic acid and simple alcohols such as methanol and ethanol, the results were similar to those shown in Figs. 8 - 9. The products of thermal degradation of the copolymers were alcohols from the acrylates (sidechain degradation), monomers (from partial depolymerization), and methacrylates of alcohols used in the synthesis of acrylates (main-chain degradation).

All earlier investigations of model acrylic PSAs were preliminary to testing of the method in research into the thermal degradation of industrial adhesives. The pyrogram of the acrylic copolymer TEC MEB-2 from ChemCycle indicates that this adhesive contains methyl-, ethyl, butyl, and 2-ethyl-hexyl moieties (Fig. 10). Acrylic acid was



Figure 5. GC of the liquid degradation products from the acrylic PSA examined

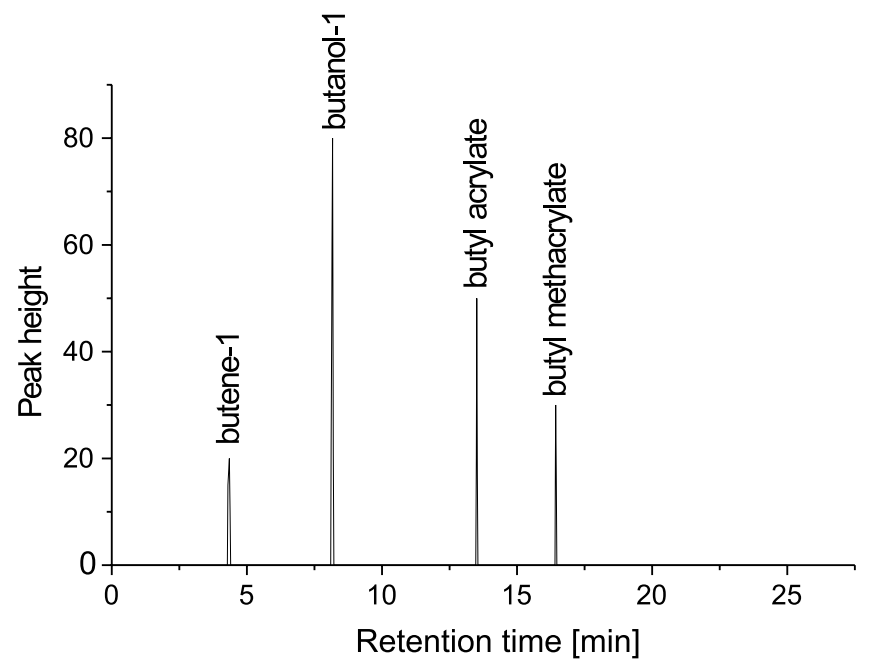

Figure 7. Pyrogram of a water-soluble copolymer synthesized from acrylic acid and butyl acrylate

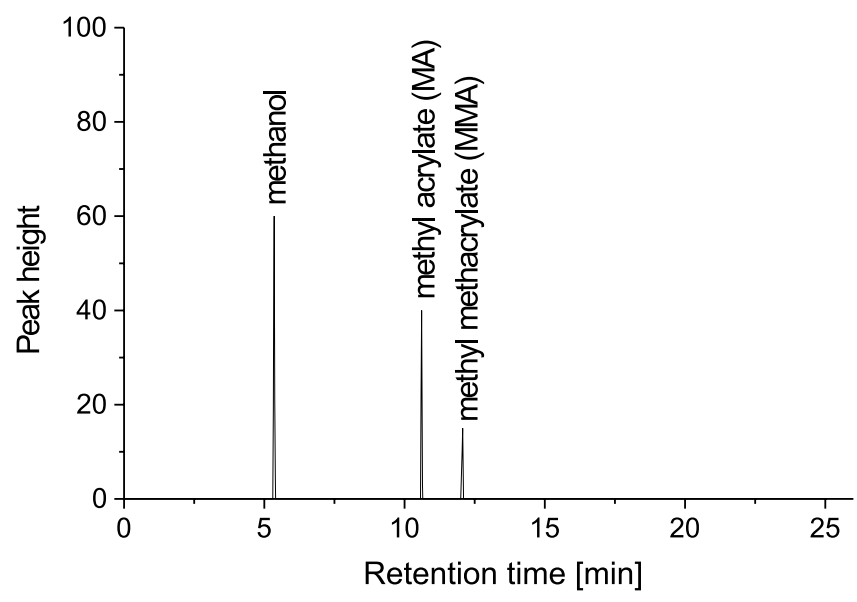

Figure 8. Chromatogram of the pyrolysate of the acrylic copolymer methyl acrylate-acrylic acid



Figure 9. Chromatogram of the pyrolysate of the acrylic copolymer ethyl acrylate-acrylic acid

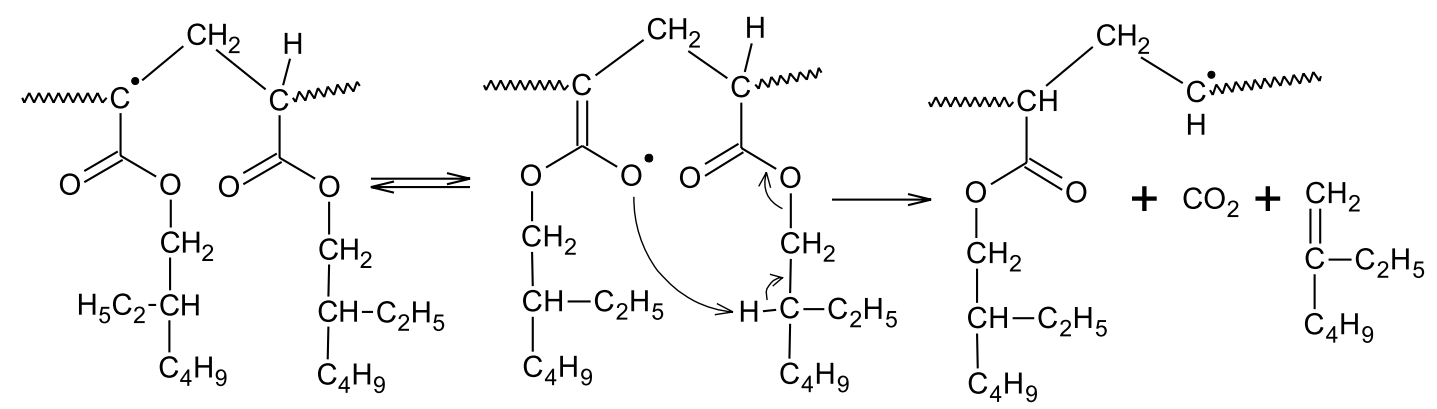

Figure 6. Thermal decomposition of acrylic PSA formed from 2-EHA and AA, with production of $\mathrm{CO}_{2}$ and olefin 
not present in the pyrolysis products. The use of Py-GC for determination of acrylic acid content in acrylic copolymers is described in reference 18 . The mechanism of formation of these compounds is explained in Fig. 11.



3. Czech, Z. \& Wesołowska, M. (2007). Development of solvent-free acrylic pressure-sensitive adhesives. European Polymer Journal. 43, 3604 - 3612. DOI: 10.1016/ j.eurpolymj.2007.05.003.

4. Haken, J.K. (1999). Pyrolysis gas chromatography of coating materials - a bibliography. Progress in Organic Coatings. 36, $1-10$.

5. Kaminsky, W. \& Eger, C. (2001). Pyrolysis of filled PMMA for monomer recovery. J. Anal. Appl. Pyrolysis. 58 $59,781-787$.

6. Sobeih, K., Baron, M. \& Gonzales-Rodriguez, J. (2008). Recent trends and developments in pyrolysis-g,as chromatography. J. Chromatogr., A. 1186, 51 - 66. DOI: 10.1016/ j.chroma.2007.10.017.

7. Ericsson, K. (2007). Thermal decomposition mechanisms common to polyurethane, epoxy, poly(diallyl phthalate), polycarbonate and poly(phenylene sulfide). J. Therm. Anal. Cal., 89, 427 - 440. DOI: 10.1007/s10973-006-8218-6.

8. Witkiewicz, Z. \& Hepter, J. (2001). Chromatografia gazowa. Warszawa, WNT.

Figure 10. Pyrogram of an acrylic copolymer containing commercial alkyl acrylates and acrylic acid

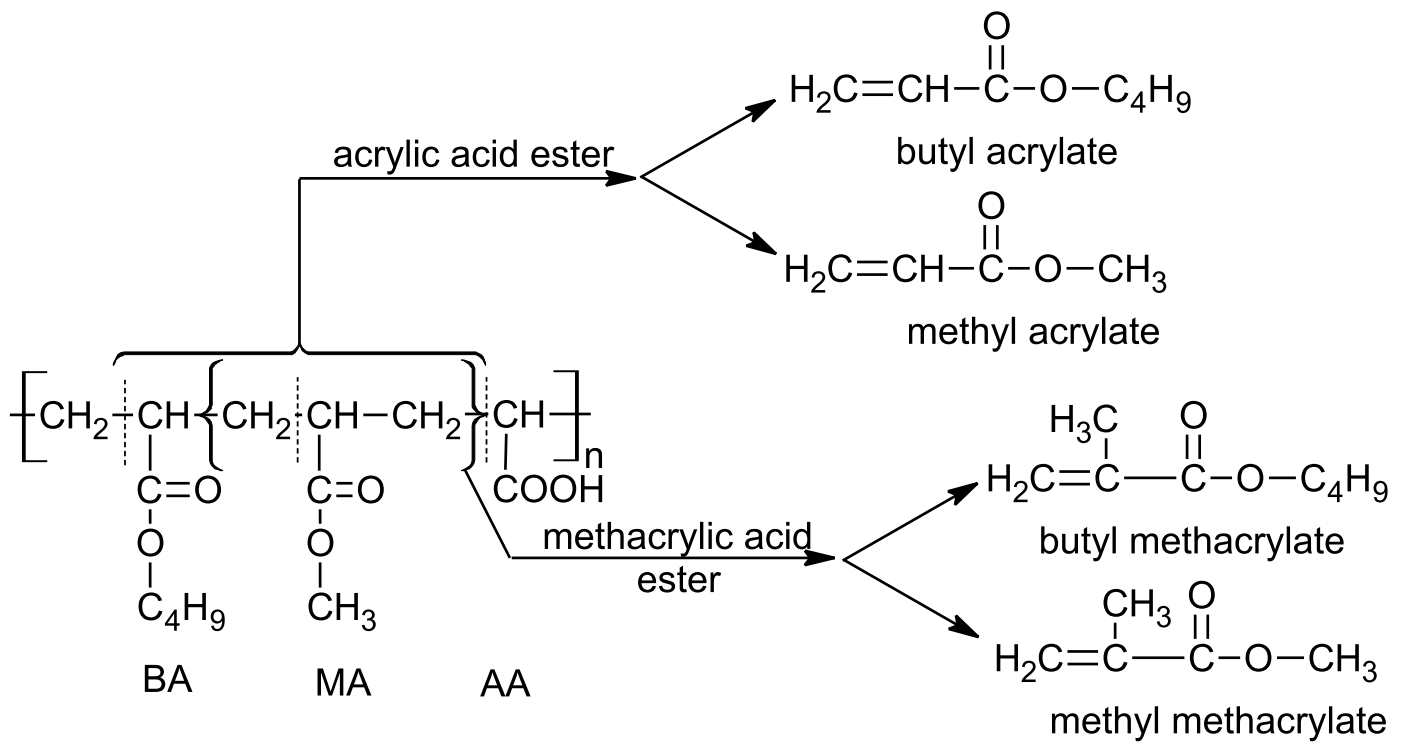

Figure 11. Formation of acrylic and methacrylic monomers during pyrolysis of acrylic copolymers

\section{CONCLUSIONS}

This review does not claim to be exhaustive, but it shows that Py-GC is an effective alternative technique for analysis of acrylic PSAs. Because the method is simple, fast, and reproducible, it is very likely that Py-GC will become one of the principal techniques in the analysis of industrial adhesives of unknown composition. The mechanism of thermal degradation of acrylic PSAs has also been described above.

\section{LITERATURE CITED}

1. Czech, Z. \& Pełech, R. (2009). The thermal degradation of acrylic pressure-sensitive adhesives based on butyl acrylate and acrylic acid. Progress in Organic Coatings. 65, 84 87. DOI: 10.1016/j.porgcoat.2008.09.017.

2. Cieślik, M. (2008). Synthesis and Application of Waterborne Polyurethane Pressure- sensitive Adhesives. Doctoral dissertation, Szczecin University of Technology, Institute of Organic Chemical Technology, Szczecin, Poland.
9. Wang, F.C.-Y. \& Meunier, D.M. (2000). Number-average molecular mass determination of polymeric material by pyrolysis-gas chromatography. J. Chromatogr. A. 888, 209 217.

10. Wang, F. C.-Y., Lohse, D.J., Chapman, B.R. \& Harrington, B.A. (2007). Determining the composition and microstructure of ethylene-propylene copolymers by pyrolysis-gas chromatography. J. Chromatogr. A. 1138, 225 - 230. DOI: 10.1016/j.chroma.2006.10.052.

11. Wang, F.C.-Y. (2000). Polymer additive analysis by pyrolysis-gas chromatography I. Plasticizers. J. Chromatogr. A. $883,199-210$.

12. Wang, F.C.-Y. (2000). Polymer additive analysis by pyrolysis-gas chromatography II. Flame retardants. J. Chromatogr. A. 886, 225 - 235.

13. Wang, F.C.-Y. \& Buzanowski, W.C. (2000). Polymer additive analysis by pyrolysis-gas chromatography III. Lubricants. J. Chromatogr. A. 891, 313 - 324.

14. Wang, F.C.-Y. (2000). Polymer additive analysis by pyrolysis-gas chromatography IV. Antioxidants. J. Chromatogr. A. 891, 325 - 336. 
15. Asperger, A., Engewald, W. \& Wagner, T. (1999). Quantitative determination of acrylate-based copolymer retarding layers on drug granules using pyrolysis-gas chromatography. J. Anal. Appl. Pyrolysis, 49, 155 - 164.

16. Lattimer, R.P., (2003). Pyrolysis of filled PMMA for monomer recovery. J. Anal. Appl. Pyrol., 68 - 69, 3 - 14, DOI: $10.1016 / \mathrm{S} 0165-2370(03) 00080-9$.

17. Tsuge, S.(1995). Analytical pyrolysis - past, present and future. J. Anal. Appl. Pyrolysis, 32, $1-6$.

18. Czech, Z. \& Pełech, R. (2008). Use of pyrolysis and gas chromatography for the determination of acrylic acid concentration in acrylic copolymers containing carboxylic groups. Polymer Testing. 27, 870 - 872. DOI: 10.1016/ j.polymertesting.2008.06.009. 